\title{
Effect of Zn substitution on magnetic properties of nanocrystalline Cobalt Chromite Muliferroic
}

\author{
Jagadish Kumar G. ${ }^{1 *}$, Durgesh Kumar ${ }^{1}$, A. Banerjee ${ }^{2}$, Chandana Rath ${ }^{1}$ \\ ${ }^{1}$ School of Materials Science \& Technology, Indian Institute of Technology, Banaras Hindu \\ University, Varanasi, 221005, India \\ ${ }^{2}$ UGC-DAE Consortium for Scientific Research, Khandwa Road, Indore, 452017, India \\ *Email: jagadishphysics@gmail.com
}

Magnetic nanoparticles have tremendous applications in various fields like high density information storage devices, sensors and magnetic drug delivery. In nanometer range, there is the competition between structural and magnetic length-scales which gives rise to a range of new magnetic phenomena, are intensively studied by basic researchers. Magnetic properties in nanosize particles are expected to be strongly affected by surface effects as it is evidenced in many ferro-, ferri- and antiferromagnetic materials. Among spinel chromites, $\mathrm{CoCr}_{2} \mathrm{O}_{4}$ is the first example of a multiferroic compound with both the spontaneous magnetization and polarization of spin origin, where the strong clamping between the ferrimagnetic and ferroelectric domains, leading to the magnetic reversal of the ferroelectric polarization, has been observed [1]. The compound undergoes a ferrimagnetic transition at $T_{c}=93 \mathrm{~K}$, and with the further lowering of temperature, the additional transition to the conical spin state, lock-in transition occur at $26 \mathrm{~K}$ and at around $15 \mathrm{~K}$ respectively [2-3]. With reducing size, we have observed an intermediate superparamagnetic phase below paramagnetic phase [4-5]. In this work, we have studied the structural and magnetic properties of $\mathrm{CoCr}_{2} \mathrm{O}_{4}$ by adding non magnetic dopant like $\mathrm{Zn}$ in Co site.

Nanoparticles of $\mathrm{Zn}$ substituted $\mathrm{CoCr}_{2} \mathrm{O}_{4}$, where $\mathrm{Zn}$ concentration is varying from 0.05 to 0.2 with an intervel of 0.05 , is synthesized by co-precipitaion technique. Stock solutions of $0.5 \mathrm{M}$ of cobalt nitrate, zinc nitrate and chromium nitrate are prepared and desired amount of chromium nitrate was taken and subjected to stirring for an hour using a magnetic stirrer. Desired quantity of cobalt nitrate and zinc nitrte was added slowly to the chromium nitrate. The mixed solution was subjected to continuous stirring at room temperature for $2 \mathrm{~h}$. Under stirring, diluted aqueous ammonia (30\%) solution was added drop wise to maintain the $\mathrm{pH}$ at 9 . The precipitate was filtered and washed several times with distilled water followed by acetone and left for drying in an oven at $120^{\circ} \mathrm{C}$ for $16 \mathrm{~h}$. We calcined the samples at $900{ }^{\circ} \mathrm{C}$ for 4 h.

The phase and crystalline structure of the calcined powders were characterized by X-ray diffraction (XRD) using an $18 \mathrm{KW}$ rotating anode $\left(\mathrm{Cu} \mathrm{K}_{\alpha}\right)$ based Rigaku powder diffractometer operating in the Bragg-Brentano geometry and fitted with a graphite monochromator in the diffracted beam. The temperature and field dependent $\mathrm{dc}$ magnetization were measured by using Physical Property Measurement System (PPMS Model no. 6000) of Quantum Design operating between $2 \mathrm{~K}$ and $350 \mathrm{~K}$. The temperature dependence a.c. susceptibility were recorded in zero external field using SQUID magnetometer at different frequencies with a.c. magnetic field $0.3 \mathrm{mT}$.

$\mathrm{XRD}$ show pure cubic phase of $\mathrm{Fd} 3 \mathrm{~m}$ space group for all samples. The crystallite size of the samples are found to be in the range of $10 \mathrm{~nm}$. Temperature dependent magnetization is measured in zero field cooled and field cooled conditions. In zero field cooled condition sample is cooled up to $2 \mathrm{~K}$ under zero applied field. Then moment of the sample is measured during heating the sample up to $300 \mathrm{~K}$. While in field cooled condition sample is cooled under 500 Oe applied field and moment is measured during subsequent heating up to $300 \mathrm{~K}$. Magnetization measurements reveal that the Curie temperature $\left(T_{c}\right)$ decreases from $87.9 \mathrm{~K}$ to $81.4 \mathrm{~K}$ with increase in ' $\mathrm{Zn}$ ' concentration from 0.05 to 0.2 as shown in Figure 1. Frequency dependent ac susceptibility confirms the spin glass behavior in $\mathrm{Zn}_{0.05} \mathrm{Co}_{0.95} \mathrm{Cr}_{2} \mathrm{O}_{4}$ sample. Microstructure dependent detail magnetic properties of these chromites are discussed.

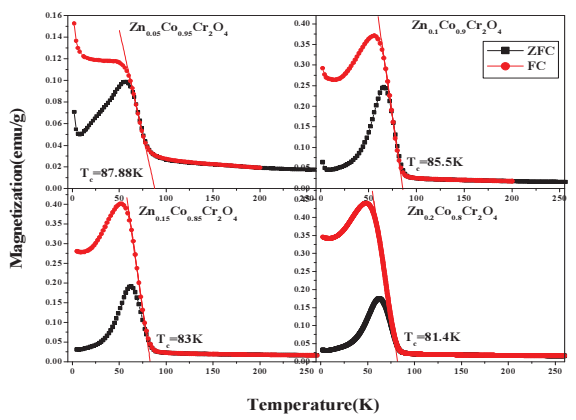

Figure 1: Temperature dependent $\mathrm{M}_{\mathrm{FC}}$ and $\mathrm{M}_{\mathrm{ZFC}}$ of $\mathrm{Zn}$ substituted $\mathrm{CoCr}_{2} \mathrm{O}_{4}$ nanoparticles

\section{References}

1. Yamasaki et al. Phy. Rev. Lett. 96 (2006) 207204.

2. Funahashi et al., J. Appl. Phys. 61 (1987) 4114.

3. Chang et al. J. Phys.: Condensed Matter 21(45) (2009) 456008

4. Chandana Rath, P. Mohanty, A. Banerjee, J. Magn. Magn. Mater. 323 (2011) 1698-1702

5. Lawrence Kumar, P. Mohanty, T. Shripathi, Chandana Rath, Nanoscience and Nanotechnology Letters Vol. 1 (2009) 199-203. 